\title{
Left Main Coronary Artery Spasm: Case Report
}

\author{
Vijay Kumar Bodicherla ${ }^{1}$ Srikrishna Srikakulapu ${ }^{1} \quad$ Lalitha Nemani $^{1}$ \\ ${ }^{1}$ Cardiology Nizams Institute of Medical Sciences, India \\ Ind J Car Dis Wom 2021;6:186-188. \\ Address for correspondence Dr Vijay Kumar Bodicherla, MD, \\ Cardiology Nizams Institute of Medical Sciences, India \\ (e-mail: Vijay.bodicherla@gmail.com).
}
Abstract
Keywords
- catheter induced
- LMCA
- spasm
- spontaneous
- vasospasm

Vasospasm of coronary artery is a well-known cause for acute coronary syndrome (ACS) but left main coronary artery (LMCA) spasm is very rare. It may be iatrogenic or spontaneous. Here we report a case of a 44-year-old female who presented with chronic stable angina and experienced LMCA vasospasm while undergoing coronary angiography.

\section{Introduction}

Coronary artery vasospasm is a well-known etiology for angina. Vasospasm of right coronary artery is generally seen but spontaneous vasospasm of ostial left main coronary artery (LMCA) is rarely seen. Vasospasm of LMCA may be iatrogenic, a complication of coronary angiography, or spontaneous. ${ }^{1}$ The junction of the aorta with the ostium of the LMCA contains high-percentage of elastic fiber, and this area has less atherosclerotic plaque burden. Hereby, we are presenting a case of LMCA ostial vasospasm which is spontaneous and spasm-relieved in subsequent angiography.

\section{Case}

A 44-year-old female presented with exertional angina and shortness of breath class II since 3 months. She was a premenopausal, nondiabetic patient. She was diagnosed as being hypertensive a month earlier. She was taking Telemisartan $40 \mathrm{mg}$ for hypertension and there was no other drug intake. There was no h/o oral contraceptive intake or family h/o of premature atherosclerosis.

On examination, vitals were normal with normotension. Clinical examination of heart and systemic examination were normal. Electrocardiogram showed normal sinus rhythm without significant ST-T changes (-Fig. 1). Twodimensional (2D) echocardiogram showed no regional wall motion abnormalities with good biventricular function. There was no mitral or tricuspid regurgitation. However, Doppler examination showed grade l diastolic dysfunction. The treadmill test was positive for inducible ischemia in stage 2 of Bruce protocol. Cardiac enzymes were normal (LDH 165 units/mL, CPK 49 units/L).

Conventional contrast coronary angiogram showed ostioproximal LMCA 80 to $90 \%$ stenosis, with proximal left anterior descending coronary artery (LAD) $40 \%$ stenosis, and right coronary artery as normal (-Fig. 2a, 2b). When diagnostic $6 \mathrm{~F}$ catheter was engaged, there was no damping and there was good reflex which was seen in - Fig. 2. Maximum narrowing of the LMCA was in proximal portion, with real ostium of LMCA being normal. The lesion was tapering toward the bifurcation, without involving the bifurcation site as shown in - Fig. 2 a, but in right anterior oblique (RAO) cranial view, the lesion looks like uniform narrowing of the whole length of LMCA (-Fig. 2b). Percutaneous coronary intervention (PCI) was planned with intravascular ultrasound (IVUS) guidance, as patient requires financial sanction from their company.

Check injection was done with 7F Judkin's left 3.5 curve before percutaneous transluminal coronary angioplasty
DOI https://doi.org/ 10.1055/s-0041-1736247. ISSN 2455-7854.

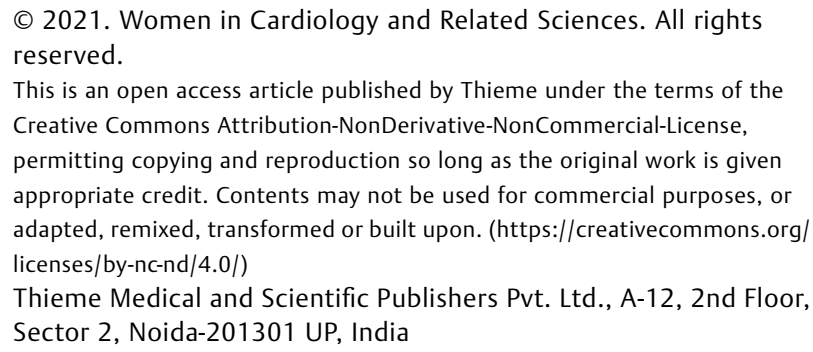

This is an open access article published by Thieme under the terms of the Creative Commons Attribution-NonDerivative-NonCommercial-License, permitting copying and reproduction so long as the original work is given appropriate credit. Contents may not be used for commercial purposes, or adapted, remixed, transformed or built upon. (https://creativecommons.org/ licenses/by-nc-nd/4.0/)

Thieme Medical and Scientific Publishers Pvt. Ltd., A-12, 2nd Floor, Sector 2, Noida-201301 UP, India 


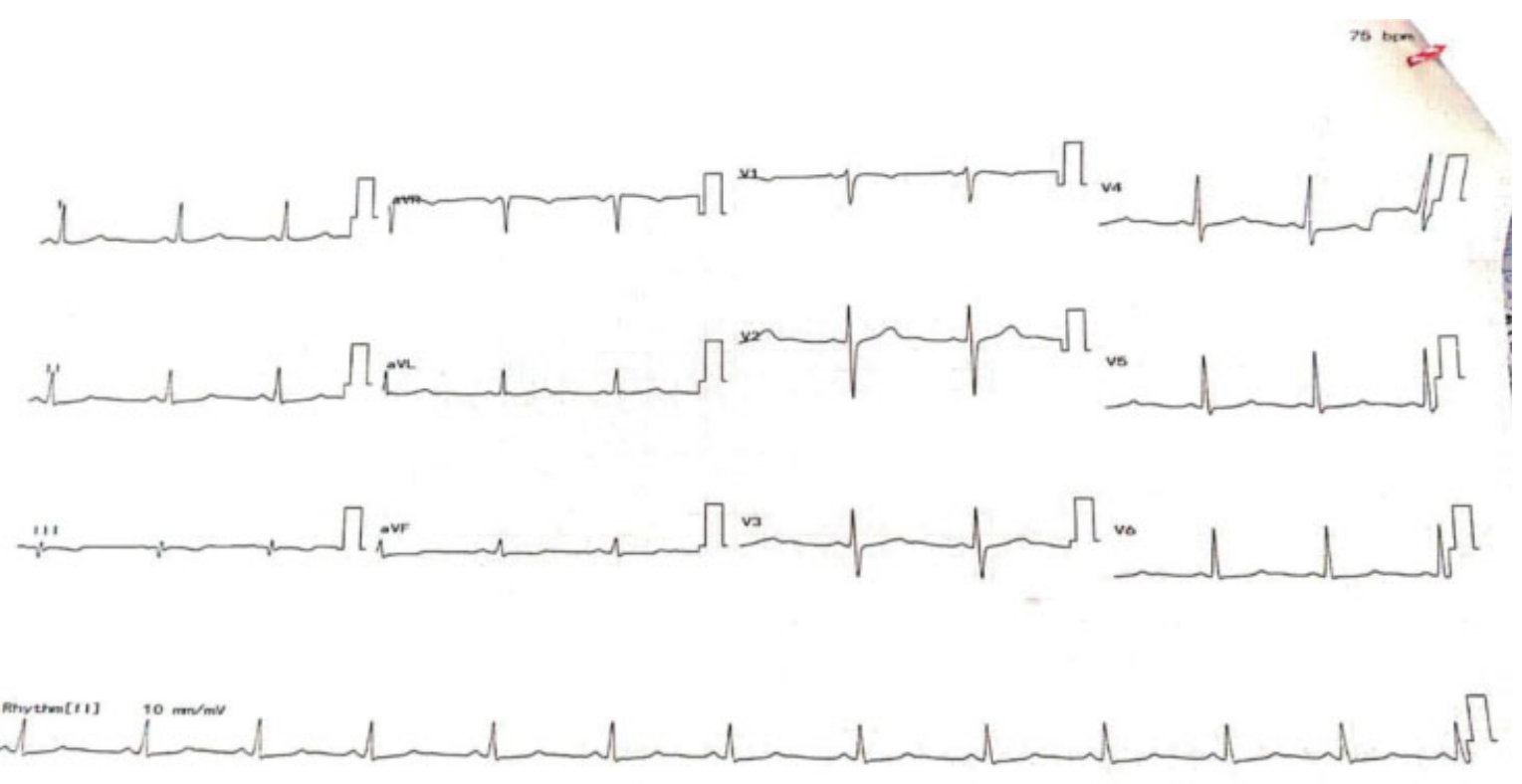

Fig. 1 Normal electrocardiogram.

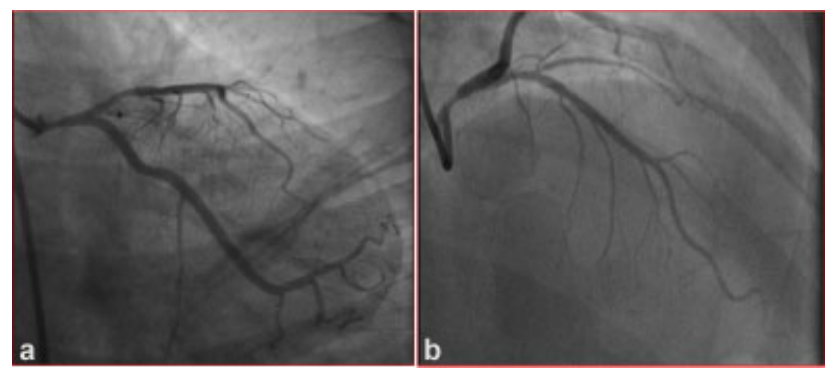

Fig. 2 (a) RAO caudal view showing LMCA ostioproximal stenosis. (b) RAO cranial view showing LMCA lesion. Abbreviations: LMCA, left main coronary artery; RAO, right anterior oblique.

(PTCA). LMCA was normal in different views and LAD lesion was significant than before. Both features were suggestive of coronary spasm. Normalization of LMCA suggests that there was completely only spasm in LMCA (-Fig. 3a, 3c), but in $\mathrm{LAD}$, there may be underlying atherosclerotic lesion super added with spasm, as both times coronary angiography (CAG) showed some degree of coronary narrowing (-Fig. 3a, 3c). Patient had class II angina with treadmill test (TMT) and showed inducible ischemia along with borderline lesion of LAD. Fractional flow reserve (FFR) for LAD was done, which showed preadenosine of 0.98 and postadenosine of 0.92 , both insignificant.

\section{Discussion}

Vasospasm of right coronary artery is a common phenomenon but LMCA spasm is relatively rare. Coronary artery spasm of the LMCA may be spontaneous (as variant angina is an uncommon cause; when performing provocative tests using acetylcholine or ergonovine) or iatrogenic (catheter- or guidewire-induced). ${ }^{1}$ Spasm because of catheter is common in right coronary artery but spasm of left coronary is quite uncommon. ${ }^{2}$ Spasm because of catheter must be considered in the diagnosis of LMCA disease, especially when angiography reveals that significant narrowing is present in LMCA only and atherosclerotic disease in other coronary arteries is not significantly present. But in our case, there was associated lesion in LAD, which is a potential cause for misdiagnosis in angiography and has an incidence between $0.3 \%$ and $2.9 \%$. Coronary CT angiography was able to unmask catheterinduced coronary spasm as a reason for a "false-positive." LM coronary stenosis in invasive angiography. CT-CAG is helpful for LMCA limited spasm before surgery, as it delineates lesions properly when it is catheter-induced, so it can

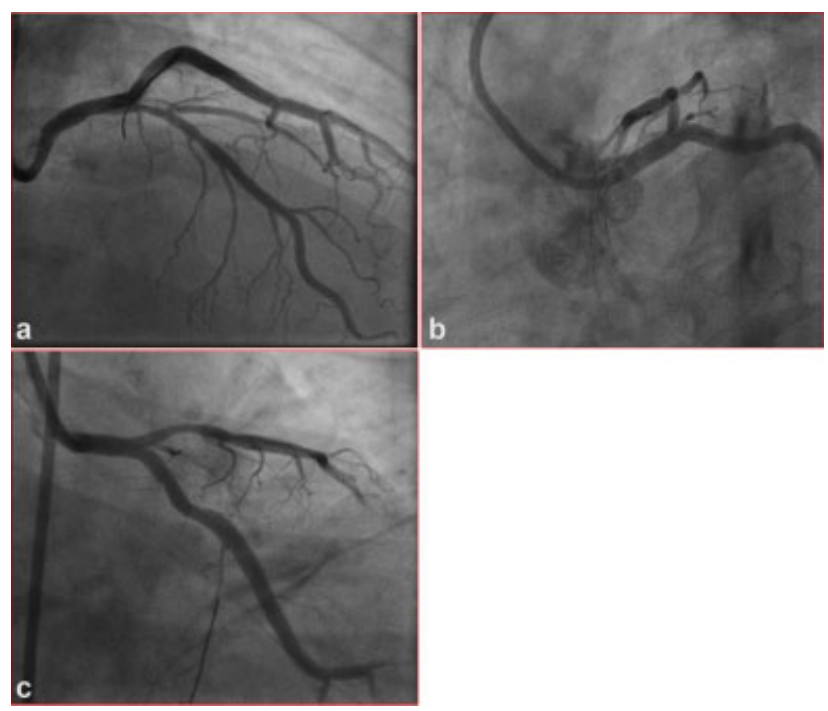

Fig. 3 (a) LMCA in RAO cranial view. (b) LMCA in LAO caudal view. (c) LMCA in LAO caudal view. Abbreviations: LMCA, left main coronary artery; LAO; left anterior oblique; RAO, right anterior oblique. 
be limited to patients with high-index of suspicion of LMCA vasospasm.

Vasospasm of the LMCA can accompany atherosclerosis or can be the solitary reason of LMCA lesions. Intracoronary nitroglycerin can be used during conventional CAG to differentiate whether a vasospasm occurs or an atherosclerotic plaque is present in LMCA. It is conceivable that certain anatomic variations in the length, caliber, course, or even origin of the LMCA may predispose it to spasm. It is not known that catheter-induced spasm predisposes to spontaneous vasospasm. ${ }^{2}$ In our case, LMCA length was $8 \mathrm{~mm}$ (not too short or loo long), and of normal origin, course and caliber. So, there are no features that predisposes to the development of the spasm.

In ostial LMCA, stenosis damping of pressure on engagement of catheter with ostium and the absence of reflux of dye into the coronary sinus on injection occurs. But similar features can happen even in the LMCA spasm. Usually, catheter-induced vasospasm will occur within $1 \mathrm{~mm}$ to the catheter rather than true spasm and usually relieved by intracoronary nitroglycerin. ${ }^{3}$ In our case, more narrowing of LMCA was seen in the proximal segment of LMCA, that is, one $\mathrm{mm}$ after the ostium with relative sparing of the ostium. May be catheter-induced spasm is superimposed. But there was more significant narrowing of the LAD in second day injection, which means that patient had vasospastic component in addition. IVUS is useful to reveal any abnormalities in the vessel wall.

Catheter-induced spasms are usually maximum at the site of the contact with the vessel. Remote spasms due to catheter as such does not happen, but catheter stimulation of the prone smooth muscle cell activation can produce spasm at remote areas. Esenboga et al reported that in patients presenting with myocardial infarction (MI) catheter-induced multiple nonproximal coronary spasms. ${ }^{4}$

Usually, atherosclerotic plaques develop at angulation and bifurcations due to shear stress; hence, ostial LMCA lesions are less frequent than mid and distal LM lesions. ${ }^{5}$ If some irregularity is seen in the lesion, then atherosclerotic lesions may be more likely. Smooth narrowing suggests more of a spasm. Spasm can be focal, diffuse, or multifocal, involving multiple coronary arteries. According to Seung-Woon Rha et al, diffuse coronary artery spasm was associated with adverse 3-year clinical outcomes. Therefore, those who show diffuse CAS should require intensive antianginal management and close clinical follow-up. ${ }^{6}$ In this case, both LMCA and LAD were involved. So, better follow this case for longterm with intensive antianginal therapy.

In conclusion, in the present case, LMCA and LAD spasm may be spontaneous variants of vasospastic angina. This can be confirmed by vasospastic testing in cath laboratory, which was not done in this case.

\section{Conclusion}

In conclusion, spasm of LMCA is a rare entity which may be spontaneous or iatrogenic and can lead to acute coronary syndromes. It is a beginner's trap, so immediate evaluation is necessary through CT CAG, IVUS, and FFR to rule out significant lesion, which can be treated medically with nitrates and calcium channel blockers. PTCA or coronary artery bypass grafting (CABG) can be based on SYNTAX score in patients who are refractory to medical therapy.

\section{Conflicts of Interest}

None declared.

\section{References}

1 Sueda S, Kohno H, Fukuda H, et al. Clinical impact of selective spasm provocation tests: comparisons between acetylcholine and ergonovine in 1508 examinations. Coron Artery Dis 2004;15(08): 491-497

2 Parakh N, Singh S, Math RS, Sharma S, Bahl VK. Catheter-induced left main coronary artery spasm. J Cardiovasc Med (Hagerstown) 2009;10(03):288-289

3 Edris A, Patel PM, Kern MJ. Early recognition of catheter-induced left main coronary artery vasospasm: implications for revascularization. Catheter Cardiovasc Interv 2010;76(02):304-307

4 Esenboga K, Baskovski E, Ozyuncu N, Tutar E. Catheter-induced multiple non-proximal coronary spasm in a patient presenting with myocardial infarction. Cureus 2020;12(03):e7456

5 Kaya M, Erhan K, Ihsan B. Ostial left main coronary artery stenosis mimics catheter-induced vasospasm: a case of emergency coronary artery bypass surgery. ICR Journal 2015;1(01):55-57

6 Rha S-W, Choi BG, Choi SY, et al. comparison of diffuse with focal coronary artery spasm in patients with vasospastic angina on 3year clinical outcomes. J Am Coll Cardiol 2014;63(12): s0735-s1097 